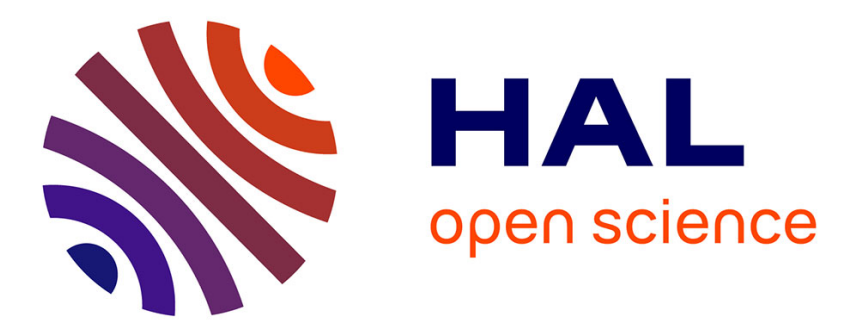

\title{
Utilisation des données SIGAPS pour mesurer l'impact du plan Alzheimer sur la production d'articles des CHU
}

\author{
Patrick Devos, Antoine Cortot, Joel Menard
}

\section{To cite this version:}

Patrick Devos, Antoine Cortot, Joel Menard. Utilisation des données SIGAPS pour mesurer l'impact du plan Alzheimer sur la production d'articles des CHU. La Presse Médicale, 2016, La presse médicale, 45, pp.E1-E10. 10.1016/j.lpm.2015.07.023 . hal-03195739v2

\section{HAL Id: hal-03195739 \\ https://hal.univ-lille.fr/hal-03195739v2}

Submitted on 2 Jun 2021

HAL is a multi-disciplinary open access archive for the deposit and dissemination of scientific research documents, whether they are published or not. The documents may come from teaching and research institutions in France or abroad, or from public or private research centers.
L'archive ouverte pluridisciplinaire HAL, est destinée au dépôt et à la diffusion de documents scientifiques de niveau recherche, publiés ou non, émanant des établissements d'enseignement et de recherche français ou étrangers, des laboratoires publics ou privés. 
Utilisation des données SIGAPS pour mesurer l'impact du Plan Alzheimer sur la production d'articles des CHU.

Measure of Plan Azheimer's impact on the University Hospitals articles production through SIGAPS data.

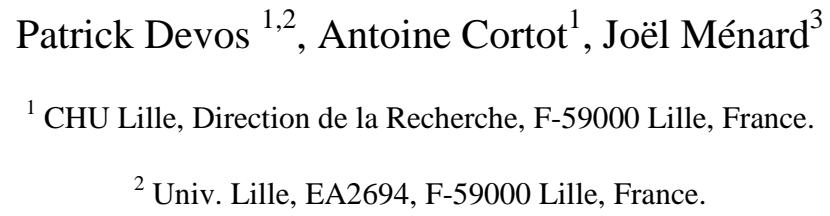

${ }^{3}$ Fondation Plan Alzheimer, 75013 Paris, France, Université Paris Descartes, Faculté de Médecine, and INSERM/AP-HP Clinical Investigation Center, Georges Pompidou Hospital, Paris, France

Correspondance : Patrick Devos, Direction de la Recherche, CHRU de Lille, 1 Av O Lambret, F-59000 Lille, France Email : patrick.devos@univ-lille2.fr

Keywords : Bibliométrie, Maladie d'Alzheimer, SIGAPS, Plan de Santé Publique

\section{Résumé :}

Introduction: Un troisième Plan Alzheimer et maladies apparentées (PA) comprenant un volet Recherche détaillé a été mis en œuvre de 2008 à 2013. Le but de ce travail a été d'évaluer l'impact du PA sur la production d'articles originaux concernant la Maladie d'Alzheimer et Maladies Apparentées (MA) avant (2004-2008) et après (2009-2013) sa mise en place.

Méthodes: Le nombre et le classement basé sur le Facteur d'Impact (FI) des articles originaux produits par les 27 Centres Hospitalo-Universitaires (CHU) de Métropole ont été évalués grâce aux données du Système d'Interrogation, de Gestion et d'Analyse des Publications Scientifiques (SIGAPS) utilisé pour attribuer une partie des crédits de Missions d'Enseignement, de Recherche, de Référence et d'Innovation (MERRI). La production scientifique relative à la MA a été comparée à celle concernant 4 pathologies contrôles, 2 neurologiques (Accidents Vasculaires Cérébraux, et maladie de Parkinson) et 2 autres (diabète et SIDA). La production a été mesurée par Centre Hospitalo-Universitaire (CHU) et par inter régions selon 7 Groupements Interrégionaux de Recherche Clinique et d'Innovation (GIRCI) en calculant leurs Indices de Spécialisation (IS). 
Résultats: Le nombre d'articles sur la MA a augmenté de 1277 à 1972 (+ 54,4 \%), le score SIGAPS de 18038 à $29309(+62,5 \%)$ et le nombre d'articles classés A et B (correspondant aux $25 \%$ des meilleures revues de la spécialité) de 430 à $846(+114,1 \%)$. Ces indicateurs ont augmenté de la même manière pour la MA et les AVC, alors que pour les 3 autres pathologies l'évolution a été variable : stagnation à un haut niveau pour la maladie de Parkinson et le SIDA et augmentation pour le diabète $(+15,5 \%)$. Les IS étaient $>1$ pour les 3 pathologies neurologiques dont la MA dans le Nord-Ouest et le Sud-Ouest Outre-Mer (SOOM). Les IS des CHU sur la MA variaient peu entre les 2 périodes, 4 établissements ayant à la fois une production et un IS élevés : Toulouse, Bordeaux, Montpellier et Lille.

Conclusion : La production et la qualité des articles originaux sur la MA par les CHU ont augmenté après la mise en place du PA en 2008. Déterminer si cette augmentation est en rapport avec l'application du volet recherche du PA reste à confirmer. La production sur la MA était inégalement répartie sur le territoire français et les établissements qui avaient déjà une recherche active avant la mise en place d'un PA renforçaient celle-ci après. Enfin ce travail illustre l'utilisation stratégique qui peut être faite de SIGAPS au-delà de son utilisation comme outil de financement.

\section{Summary}

Introduction: A third Alzheimer and related diseases Plan (AP) including a detailed research part has been implemented from 2008 to 2013 . The aim of this study was to assess the AP impact on the original articles production concerning Alzheimer and related disease (AD) before (2004-2008) and after its implementation (2009-2013).

Methods: Number and Impact Factor (IF) based ranking of original articles coming from French University Hospitals (CHU) have been assessed using the data provided by the SIGAPS bibliometric software used so far for calculating allocation of the governmental medical research funding (MERRI). Scientific production on $\mathrm{AD}$ has been compared to four other control pathologies: 2 neurologic (stroke and Parkinson disease) and 2 non neurologic (diabetes and AIDS). 
Publication trends of the $27 \mathrm{CHU}$ and of the 7 Interregional Grouping of Clinical Research and Innovation (GIRCI) have been analyzed using Specialization Indices (SI).

Results: The number of AD articles increased from 1277 to $1972(+54.4 \%)$ as well as SIGAPS score from 18038 to 29309 (+62.5\%). Meanwhile number of articles rated A and B (published in the $25 \%$ best journals of the specialty) increased from 430 to $846(+114.1 \%)$. The figures for these indicators evolved similarly for $\mathrm{AD}$ and stroke while quite differently for the 3 other diseases: plateauing at a high level for Parkinson disease and AIDS and modestly increasing for diabetes. SI was $>1$ for the 3 neurologic diseases including AD in North-Western and South-West GIRCI. SI of CHU on AD did not vary before and after AP implementation, 4 CHU having both a production and an SI at a high level: Toulouse, Bordeaux, Montpellier and Lille.

Conclusion: The number and "quality" of original articles on AD production by the CHU increased after the AP starting of AP initiation in 2008. Whether or not this was directly due to the financial support provided by the AP remains to be determined. The scientific production on AD was unequally distributed throughout the country, the CHU being already strongly involved in AD research before the AP reinforced their commitment after. This work illustrates the potential implication of SIGAPS as a strategic tool for research policy. 
Ce qui était connu :

- En France, la maladie d'Alzheimer a fait l'objet de trois plans nationaux de santé publique : en 2001, 2004 et 2008 .

- SIGAPS, logiciel de bibliométrie, a été déployé dans plus de 150 établissements de Santé dans le cadre des financements MERRI.

- L'interrogation nominative et la validation par les chercheurs assurent une grande fiabilité des données issues de SIGAPS.

Ce qu'apporte l'article :

- Des données objectives et comparatives sur la production scientifique liée à la maladie d'Alzheimer avant et après la mise en place du versant recherche du 3ème plan Alzheimer.

- Un exemple des perspectives sur l'évaluation des plans de Santé Publique et l'utilisation stratégique des données SIGAPS. 
En 2008, la France mettait en place un 3ème plan national de lutte contre la maladie d'Alzheimer (MA) et les maladies apparentées [1] réalisé jusqu'en 2013. Contrairement aux deux précédents plans Alzheimer (PA) de 1999-2003 et 2004-2008, ce $3^{\text {ème }}$ se caractérisait par un soutien financier dédié à la recherche et fléché sur 14 thèmes détaillés, soutien beaucoup plus élevé que dans les deux précédents : $150 \mathrm{~K} €$ (1999), $275 \mathrm{~K} €$ (2004) et $200 \mathrm{M} €$ (2008) [2,3,4]. En 2014, il est apparu indispensable d'évaluer l'impact de cet effort important sur la production scientifique française sur la MA. Ce projet nécessitait d'avoir à disposition les outils bibliométriques. Avant le lancement du PA, des analyses bibliométriques préliminaires sur les données du Web of Science (WoS) et de la base SIGAPS (Système d'Interrogation, de Gestion et d'Analyse des Publications Scientifiques) avaient été faites par l'INSERM et le CHU de Lille, afin de repérer les CHU et les équipes (Établissements Publics à caractère Scientifique et Technologique EPST et/ou Université) déjà impliquées dans la recherche sur la MA. Une évaluation plus systématique de la production scientifique à partir des données de la base SIGAPS a été faite sur les 27 CHU de métropole avant (2004-2008) et après (2009-2013) le 3ème PA afin de détecter son éventuel impact.

Initialement ciblée sur les EPST [5,6], l'évaluation de la production scientifique s'est étendue aux Universités grâce aux informations bibliométriques fournies par l'Observatoire des Sciences et des Techniques (OST) [7], et par le Haut Conseil de l'Evaluation de la Recherche et de l'Enseignement Supérieur (HCERES) [8]. En raison de leur vocation universitaire, les CHU sont aussi appelés à évaluer leur production scientifique.

Notre but était de présenter les données de production scientifique des CHU sur la MA à partir de la base nationale SIGAPS et notamment :

- d'analyser la production des articles originaux sur la MA de 2004 à 2013 en comparant les périodes 2004-2008 (avant la mise en place du PA) et 2008-2013 (après le PA)

- de comparer cette production à celle de 4 pathologies contrôles pendant les mêmes périodes : Accidents Vasculaires Cérébraux (AVC), maladie de Parkinson, diabète et SIDA 
- d'en évaluer la répartition pour les 5 maladies entre les 7 GIRCI (Groupements Interrégionaux de Recherche Clinique et d'Innovation) et les 27 CHU métropolitains.

\section{Méthodes}

\section{a) Constitution et analyse de la base nationale SIGAPS}

Le CHU de Lille a développé SIGAPS $[9,10]$ basé sur la validation par les chercheurs des publications identifiés dans Pubmed [11], ce qui lui a permis de constituer dès 2004 un corpus fiable de son activité scientifique. Cette démarche est alors apparue complémentaire des indicateurs retenus par le Ministère de la Santé pour attribuer le financement des Missions d'Enseignement, de Recherche, de Référence et d'Innovation (MERRI) [12] parmi lesquelles figure un indicateur de publications. Le CHU de Lille a eu en 2006 « une mission consistant à assurer la diffusion et l'utilisation du logiciel SIGAPS au sein de l'ensemble des établissements hospitalo-universitaires et à permettre la définition d'un indicateur fiable du niveau de la production scientifique de ces établissements », mission élargie aux Centres de Lutte Contre le Cancer (CLCC) et aux Centres Hospitaliers ayant une activité de recherche.

En raison des enjeux financiers, on dispose donc depuis 2008, pour les établissements de santé émargeant à la répartition des crédits MERRI (837 M€ en 2014), de données SIGAPS validées et exhaustives. Les établissements exportent annuellement leurs données vers la base nationale SIGAPS qui reçoit la liste des auteurs des publications identifiés par leur PMID (PubMed IDentifier) et de leur position dans les publications. Celles-ci sont vérifiées dans PubMed, les articles pré-référencés non publiés ou rétractés sont retirés et leur type (article, lettre, éditorial, ...) est validé.

SIGAPS permet d'obtenir d'autres informations sur la recherche: thématiques identifiées par la liste des termes MeSH (pour Medical Subjects Headings) [13], niveau de publication à partir des Facteurs d'Impact (FI) des revues, répartition sur le territoire national et évolution dans le temps. 


\section{b) Catégorisation des revues dans SIGAPS}

Chaque publication est classée selon le Facteur d'Impact (FI) de la revue [14], nombre de citations, pour une année donnée, des articles publiés dans la revue au cours des deux années précédentes, divisé par le nombre d'articles publiés par la revue pendant la même période [15,16]. Dans SIGAPS, les revues d'une discipline sont classées selon leur FI décroissant en 4 quartiles, le quartile supérieur différenciant les $10 \%$ des revues ayant les FI les plus élevés (catégorie A) et les $15 \%$ des revues suivantes (catégorie B). Les quartiles suivants comprennent par FI décroissant les catégories C, D et E (25\% des revues d'une discipline au plus faible FI). Toutes les revues référencées Pubmed qui ne sont pas recensées dans le Journal of Citation Report (JCR) et qui n'ont donc pas de FI sont classées NC (Non Classée).

$$
\mathrm{FI}_{2013}=\frac{\text { Nombre de citations,en 2013,des articles parus en } 2011 \text { et } 2012}{\text { Nombre d'articles parus en } 2011 \text { et } 2012}
$$

c) score SIGAPS : pour chaque article, un score a été calculé selon la catégorie de la revue et la position des auteurs (tableau I). Le score d'un chercheur correspond à la somme des scores de tous ses articles. Le score d'une entité (service, équipe, établissement, GIRCI, ...) est la somme des scores de tous les articles des chercheurs rattachés à cette entité, chaque article n'étant comptabilisé qu'une seule fois en prenant en compte le meilleur score.

L'analyse a été réalisée sur les données des 27 CHU de France Métropolitaine pour la période 20042013 (dernier export SIGAPS en novembre 2014). L'évolution du nombre de publications sur la MA a été comparée à celle de 4 autres pathologies « contrôles », 2 neurologiques (AVC et maladie de Parkinson) et 2 non-neurologiques (diabète et SIDA). Leur répartition sur le territoire national a été analysée pour les 7 GIRCI et les 27 CHU de la métropole. Le GIRCI Sud-Ouest-Outre-Mer (SOOM) n'a été analysé que sur les CHU de métropole et pas ceux d'outre-Mer. 
d) Indice de spécialisation (IS) : c'est le rapport du pourcentage d'articles (ou du score) d'une pathologie donnée pour une institution donnée sur le pourcentage d'articles (ou du score) de la même pathologie au niveau national [17]. Par exemple, pour le CHU de Lille, de 2004 à 2008, le score MA a été de 1644 pour un global de 48553, soit 3,39 \%. Sur la même période, au niveau national, le score MA a été de 18038 pour un global de 984350, soit 1,83\%. L'IS du CHU de Lille, dans la MA, pour la période 2004-2008 a donc été de 3,39/1,83, soit 1,85. Un IS > 1 indique une surspécialisation dans un thème de recherche alors qu'un IS $<1$, indique une sous-spécialisation.

La liste des requêtes utilisées dans la base PubMed pour délimiter le périmètre de recherche des publications sur la MA et les 4 autres pathologies est en annexe 1. N'ont été pris en compte que les articles originaux («journal article »).

f) Analyse statistique : La distribution des IS ne suivant pas une loi normale (test de Shapiro-Wilk), les résultats ont été présentés par la médiane, minimum et maximum. Les corrélations entre IS selon les 2 périodes ont été estimées à l'aide du coefficient de corrélation de Spearman.

\section{$\underline{\text { Résultats }}$}

a) Production d'articles originaux

Sur les 3208248 articles répertoriés dans Pubmed entre 2004 et 2008, 68892 (2,15\%) ont impliqué au moins un chercheur des CHU contre 4206147 publiés entre 2009 et 2013, 81900 articles $(1,95 \%)$, soit une baisse de 9,3\% de la contribution des CHU dans la recherche mondiale. A l'opposé, les publications sur la MA $(+5,0 \%)$ et les AVC $(+3,3 \%)$ ont augmenté, contrastant avec une baisse de celles sur la maladie de Parkinson (- 12,9 \%), le diabète (- 10,9\%) et le SIDA ($17,2 \%)$ (Tableau II). 
De 2004 à 2008, 1277 articles sur la MA ont été publiés, totalisant un score SIGAPS de 18038 contre 1972 de 2009 à 2013, totalisant un score de 29 309, soit $+54,5 \%$ pour le nombre d'articles et $+62,5 \%$ pour le score. Ce dernier chiffre illustre l'augmentation du nombre d'articles classés A et B de 430 à $846(+114,1 \%)$ (Figure 1). Le nombre d'articles, le score SIGAPS et le pourcentage d'articles A et B sur la MA et les 4 pathologies contrôles ont augmenté de manière proche pour la MA et les AVC, alors que pour les 3 autres pathologies l'évolution a été variable : stagnation à un haut niveau pour la maladie de Parkinson et le SIDA, augmentation pour le diabète (Tableau III).

\section{b) Répartition géographique}

Les IS dans les 7 GIRCI sur les 5 pathologies et pour les 2 périodes sont dans la Figure 2 et le Tableau IV. Les IS ont été > 1 pour les 3 pathologies neurologiques dont la MA dans le NordOuest et le SOOM : 1,73 dans le Nord-Ouest et 1,94 dans le SOOM de 2004 à 2008 et 1,54 et 2,19 de 2009 à 2013. Les IS du diabète et du SIDA ont également été > 1 dans le SOOM alors que dans le Nord-Ouest l'IS du SIDA a été $<1$ pendant les 2 périodes, quand celui du diabète est passé de 0,95 à 1,25. Dans les autres GIRCI, l'IS dans le Sud Méditerranée a été > 1 pour la MA alors que cette région a plutôt été sous spécialisée dans les 4 autres pathologies sauf le SIDA. Dans les 4 autres GIRCI, les IS sur la MA ont été < 1 pendant les 2 périodes, en particulier en Ile de France qui par contre a été sur spécialisée sur le SIDA (1,43 et 1,51). La production de l'AP/HP a représenté de 2004 à 2013, 28,3 \% des articles sur la MA produits par les CHU, 38,9 \% de celle sur les AVC, $27 \%$ de la maladie de Parkinson, 37,9\% du diabète et 48,4\% de la production sur le SIDA.

Sur les figures 3 et 4, les IS des CHU sur la MA et les 4 pathologies contrôles entre les 2 périodes sont représentés ainsi que la contribution de chacun d'eux sur chaque pathologie. La taille de la bulle est proportionnelle au score SIGAPS total sur 2004-2013, permettant d'apprécier simultanément la contribution à la production nationale et la spécialisation. Hormis pour Angers, les 
IS sur la MA ont peu varié entre les 2 périodes (corrélation entre les IS avant et après : $r=0,79$ ). Quatre établissements ont eu à la fois une production et un IS élevés : Toulouse, Bordeaux, Montpellier et Lille. Caen a eu un IS élevé avec une production globale de moitié de celle de chacun des 4 précédents (figure 3).

Dijon et Lille ont eu une production et des IS élevés sur les AVC, et, comme pour la MA, Caen a augmenté son IS. En ce qui concerne la maladie de Parkinson, 4 CHU ont eu une production et des IS élevés: Grenoble, Toulouse, Clermont-Ferrand et Lille avec une forte surspécialisation de Grenoble (IS > 4). L'AP-HP (Assistance Publique - Hôpitaux de Paris) a représenté à elle seule une part importante de la production scientifique, mais avec des IS variables : < 1 pour la MA et la maladie de Parkinson, autour de 1 pour les AVC et le diabète, 1,5 pour le SIDA. Les IS des pathologies contrôles ont été fortement corrélés entre les 2 périodes, (r compris entre 0,67 -0,90) (figure 4).

\section{$\underline{\text { Discussion }}$}

Nous avons mis en évidence une augmentation du nombre (+ $50 \%)$ d'articles originaux sur la MA produits par les CHU français pendant le $3^{\text {ème }} \mathrm{PA}$.

Afin d'interpréter cette évolution, il faut la comparer à celles de 4 autres pathologies «contrôles » La production d'articles sur les AVC (+35\%), a été après la MA (+ $54 \%)$, celle qui augmentait le plus. Pour la maladie de Parkinson, l'augmentation (+ $17 \%$ ) a été comparable à celle des articles sur le diabète $(+15 \%)$. Quant au SIDA, le nombre d'articles a peu varié $(+1,4 \%)$ entre les deux périodes.

Les progressions les plus fortes, aussi bien quantitatives que qualitatives, sont donc survenues pour la MA puis les AVC, deux pathologies qui recevaient le soutien d'un Plan national de lutte (20102014 pour les AVC) [18]. Le nombre de publications sur la maladie de Parkinson était la moitié de celles sur la MA mais d'un niveau initial plus élevé ce qui peut expliquer, en partie, que sa 
progression ait été moindre. Quant au SIDA et au diabète, ils étaient déjà anciennement soutenus par les autorités de Santé pour la première et faisait l'objet de recherches depuis longtemps pour la seconde en raison de l'augmentation de son incidence et de sa prévalence.

Il est difficile d'interpréter l'association entre les flux de publications et la mise en œuvre d'un Plan de Santé Publique, mais l'augmentation parallèle de celles sur la MA et les AVC, pathologies qui ont reçu le soutien d'un plan, est en faveur d'une causalité. L'évolution des données SIGAPS après 2013, prendra mieux en compte des recherches démarrées en cours de PA (cohorte MEMENTO, Centre Automatique de Traitement de l'Image, Banque Nationale Alzheimer, Centre de malades jeunes) et les efforts de formation (Diplôme Universitaire « Méthodes de développement industriel : maladie d'Alzheimer et maladies apparentées ») pour confirmer une association non fortuite. Une autre analyse d'impact du $3^{\text {ème }}$ PA a été réalisée sur les données WoS pour la période 2003-2012 [19] et ses résultats étaient concordants avec ceux de SIGAPS pour 2004-2013. La comparaison du WoS et de SIGAPS sur les mêmes périodes et les mêmes requêtes, pourrait être évaluée.

Les CHU déjà investis dans la recherche sur la MA dans les 5 ans précédant le 3ème PA ont été ceux qui ont le plus produit en seconde période, les équipes déjà impliquées avant captant le plus de financements après. L'analyse a considéré l'AP-HP dans sa globalité comme un seul CHU, mais des résultats plus instructifs auraient pu être obtenus, en particulier sur les IS, si elle avait été faite à un niveau plus fin (Groupements Hospitaliers et/ou Universités par exemple). En plus des informations sur le nombre d'articles (et leur répartition A, B, C, D, E), les données SIGAPS ont permis de repérer des établissements surspécialisés sur la MA, d'analyser les domaines sur lesquels portaient les publications, mais aussi d'identifier les recherches associées et les collaborations.

Ce travail repose sur SIGAPS, conçu pour analyser l'activité de recherche du CHU de Lille, et utilisé depuis pour la répartition nationale des crédits MERRI. Il illustre l'utilisation plus 
stratégique que financière qui peut être faite de SIGAPS, ce qui renforce la nécessité d'en connaitre les limites méthodologiques.

Bien que SIGAPS soit jugé robuste [20], peu de travaux d'évaluation scientifique ont été réalisés à partir de ses données [21-25]. L'interrogation nominative de PubMed, et non sur les adresses peu fiables (2700 variantes identifiées pour le CHU de Lille entre 1990 et 2014), et la validation des publications par les chercheurs eux-mêmes, sont les deux piliers méthodologiques qui assurent la fiabilité des données SIGAPS d'un chercheur, d'un établissement ou d'une inter région.

L'une des limites de SIGAPS est qu'il ne comptabilise que les personnels hospitaliers et hospitalouniversitaires, en omettant les titulaires des EPST (INSERM, CNRS, INRA ...) ou de l'Université, ce qui sous-estime le poids de la recherche fondamentale et translationnelle. Cette sous-évaluation est minimisée par l'appartenance d'un grand nombre de PU-PH aux équipes labellisées par les EPST. D'ailleurs l'analyse parallèle faite à partir du WoS révèle que l'on retrouve dans SIGAPS 52 $\%$ des articles originaux français identifiés dans le WoS [26].

La délimitation du périmètre de recherche pour une pathologie dans PubMed est un autre facteur limitant potentiel, les résultats étant dépendants de la requête utilisée pour l'extraction. Pour la MA, nous avons utilisé successivement 3 requêtes: Alzheimer's Disease, Dementia et une requête élargie (annexe 1). Quel que soit le nombre d'articles identifiés d'une requête à l'autre, les mêmes tendances ont été observées. Afin de limiter les risques «d'empiètement» sur le territoire d'autres pathologies, il est nécessaire, de faire valider les requêtes et de vérifier, comme cela a été fait ici, le contenu des articles originaux obtenus pour s'assurer de l'adéquation des articles à la requête et éventuellement améliorer celle-ci.

Ce travail porte sur les 5 ans qui ont suivi le $3^{\text {ème }} \mathrm{PA}$, délai court pour identifier des évolutions au regard de la durée de certaines études, de la lenteur des procédures réglementaires et financières de leur mise en œuvre, et des choix des jurys. Une mesure continue de la production scientifique, avec SIGAPS et le WoS, faciliterait les décisions des jurys en réponse aux appels d'offres par l'accès 
immédiat des experts à la production scientifique validée et transparente. Elle permettrait également d'orienter les choix stratégiques des universités, des EPST et des hôpitaux, et aussi de valoriser les équipes émergentes, voire même d'adapter les politiques de recherche en santé aux nouvelles régions.

Nous avons mis en évidence une augmentation de la production d'articles originaux sur la MA par les CHU après la mise en place du $3^{\text {ème }}$ PA en 2008. Déterminer si celle-ci est en rapport avec le PA reste à confirmer par une analyse plus longue (à la fois par SIGAPS et le WoS), au moins encore pendant quelques années pour prendre en compte les études non encore publiées. La production sur la MA était inégalement répartie en France, mais les établissements qui avaient déjà une recherche active avant la mise en place d'un PA renforçaient celle-ci après. L'utilisation stratégique de SIGAPS va très au-delà de son utilisation comme outil de financement de la part recherche des MERRI.

\section{Conflits d'intérêts}

Les auteurs n'ont aucun conflit d'intérêt à déclarer.

Remerciements : les auteurs remercient Ghislaine Filliatreau (OST) pour la relecture de ce manuscrit et ses conseils qui nous ont permis de l'améliorer. Merci également aux relecteurs pour leurs remarques pertinentes.

Contributions : Patrick Devos a réalisé les extractions et analyses de données. Patrick Devos, Antoine Cortot et Joël Ménard ont participé au choix des questions posées, à l'interprétation des résultats et à la rédaction de l'article. 


\section{Légende des figures :}

Figure 1: Evolution quantitative et qualitative du nombre d'articles originaux sur la maladie d'Alzheimer (MA) publiés par les CHU de 2004 à 2013 (données SIGAPS)

Figure 2: Indices de Spécialisation (IS) de la production scientifique des inters régions françaises sur 5 pathologies de 2004 à 2008 et 2009 à 2013. En bleu, l'IS sur la période 2004-2008, en jaune sur la période 2009-2013.

Figure 3 : Comparaison des Indices de Spécialisation (IS) «Alzheimer » des CHU métropolitains entre les 2 périodes 2004-2008 et 2009-2013. La taille de la bulle est proportionnelle au score SIGAPS global des publications sur la maladie d'Alzheimer (MA) sur la période 2004-2013.

Figure 4 : Comparaison des Indices de Spécialisation (IS) AVC, Parkinson, Diabète et Sida des CHU métropolitains entre les 2 périodes 2004-2008 et 2009-2013. La taille de la bulle est proportionnelle au score SIGAPS global des publications sur chacune de ces maladies sur la période 2004-2013.

\section{Références}

[1] Spinney L. Alzheimer's disease funding and the French health system Lancet 2008;7:26-27.

[2] http://www.sante.gouv.fr/IMG/pdf/plan_2001_2005.pdf (accès le 09-09-2015)

[3] http://www.sante.gouv.fr/IMG/pdf/planalzheimerbrochure.pdf (accès le 09-09-2015)

[4] http://www.plan-alzheimer.gouv.fr/ (accès le 09-09-2015)

[5] https://www.eva2.inserm.fr/EVA/jsp/Bibliometrie/presentation_biblio.jsp (accès le 09-092015)

[6] http://lalist.inist.fr/?tag=bibliometrie (accès le 09-09-2015)

[7] http://www.hceres.fr/ (accès le 09-09-2015)

[8] http://www.obs-ost.fr/frindicateur/analyses_et_indicateurs_de_reference (accès le 09-092015)

[9] Devos P, Dufresne E, Renard JM, Beuscart R. SIGAPS: a prototype of bibliographic tool for medical research evaluation. Stud Health Technol Inform 2003;95:721-6.

[10] Devos P, Lefranc H, Dufresne E, Beuscart R. From bibliometric analysis to research policy: the use of SIGAPS in Lille University Hospital. Stud Health Technol Inform 2006;124:543-8.

[11] http://www.ncbi.nlm.nih.gov/pubmed/ (accès le 09-09-2015)

[12] http://www.sante.gouv.fr/les-missions-d-enseignement-de-recherche-de-reference-et-dinnovation-merri.html (accès le 09-09-2015) 
[13] http://www.nlm.nih.gov/mesh/meshhome.html (accès le 09-09-2015)

[14] Garfield E. Citation analysis as a tool in journal evaluation. Science 1972, 178:471-479.

[15] Seglen PO, Why the impact factor of journals should not be used for evaluating research. British Medical Journal 1997314:498-502.

[16] Moed, HF. The impact-factors debate: the ISI's uses and limits. Nature 2002; 415(6873), 731732

[17] http://www.obsost.fr/sites/default/files/Note_pratique_RappelsIndicateursBibliometriques_janvier2014.pdf (accès le 09-09-2015)

[18] http://www.sante.gouv.fr/le-plan-national-d-actions-avc-2010-14.html (accès le 09-09-2015)

[19] Devos P, Haeffner-Cavaillon N, Ledoux S, Balandier C, Ménard J. Assessing the French Alzheimer plan. Lancet 2014 May 24;383(9931):1805

[20] Darmoni SJ, Ladner J, Devos P, Gehanno JF. Robustesse du score SIGAPS, critère bibliométrique pour valoriser les publications des établissements de santé. Presse Med. 2009 Jul-Aug;38(7-8):1056-61.

[21] Rabineau D, Dhainaut JF. Étude comparative des facultés de médecine en France : premiers résultats. Presse Med. 2010 Jan;39(1):e1-10.

[22] Baufreton C, Chrétien JM, Moreau-Cordier F, Moreau F, Portefaix H, Branchereau H, et al. La production scientifique issue de la formation initiale à la faculté de médecine d'Angers entre 2002 et 2008 : de bonne qualité mais insuffisant. Presse Med. 2012 May;41(5):e213-9.

[23] Rouprêt M, Drouin SJ, Faron M, Glanard A, Bitker MO, Richard F, et al. Analyse des scores SIGAPS des services de chirurgie au sein des hôpitaux universitaires de l'Assistance publique-Hôpitaux de Paris : quelle est la place de l'urologie? Prog Urol. 2012 Mar; 22(3):182-8.

[24] Ruffion A, Descotes JL, Kleinclauss F, Zerbib M, Dore B, Saussine C, Lechevallier E. Le score Sigaps est-il un mode d'évaluation pertinent pour les services universitaires ? Prog Urol. 2012 Mar;22(3):195-6.

[25] Lefèvre JH, Faron M, Drouin SJ, Glanard A, Chartier-Kastler E, Parc Y, et al. Évaluation comparative et objective de la production scientifique des services hospitaliers au sein de l'Assistance publique-Hôpitaux de Paris : analyse des scores SIGAPS. Rev Med Interne. 2013 Jun; 34(6):342-8.

[26] Haeffner-Cavaillon N, Devos P, Ledoux S, Balandier C, Ménard J. The Third French Alzheimer Plan: analysis of the influence of a national public health initiative on scientific research productivity and impact. Alzheimer's Research \& Therapy - Sous presse. 
Tableau I : Calcul du score SIGAPS : catégorie en fonction du Facteur d'Impact (FI) de la revue et de la position dans la liste des auteurs signataires. (Ex : score d'un $2^{\text {ème }}$ auteur dans une revue $\mathrm{B}=3$ x $6=18)$

\begin{tabular}{|c|c|}
\hline \multicolumn{2}{|c|}{ Catégorie } \\
\hline A & 8 \\
\hline B & 6 \\
\hline C & 4 \\
\hline D & 3 \\
\hline E & 2 \\
\hline NC & 1 \\
\hline
\end{tabular}

\begin{tabular}{|l|c|}
\hline \multicolumn{2}{|c|}{ Position } \\
\hline $1^{\text {er }}$ auteur & 4 \\
\hline $2^{\text {nd }}$ auteur & 3 \\
\hline $3^{\text {eme }}$ auteur & 2 \\
\hline Position intermédiaire (k) & 1 \\
\hline Avant dernier auteur (ADA) & 3 \\
\hline Dernier auteur (DA) & 4 \\
\hline
\end{tabular}

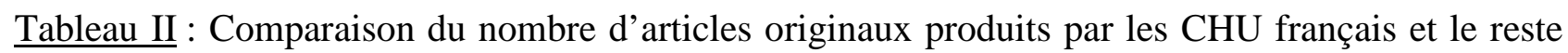
du monde sur 5 pathologies (maladie d'Alzheimer, AVC, maladie de Parkinson, diabète et SIDA) en 2004-2008 et 2009 -2013

\begin{tabular}{|c|c|c|c|c|c|c|c|}
\hline \multirow[b]{2}{*}{ Pathologie } & \multicolumn{3}{|c|}{$\begin{array}{c}\text { 2004-2008 } \\
\text { Nombre d'articles }\end{array}$} & \multicolumn{3}{|c|}{$\begin{array}{c}2009-2013 \\
\text { Nombre d'articles }\end{array}$} & \multirow[b]{2}{*}{$\begin{array}{c}\text { Evolution } \\
\%\end{array}$} \\
\hline & Pubmed & $\begin{array}{l}\text { SIGAPS } \\
27 \text { CHU }\end{array}$ & Part (\%) & Pubmed & $\begin{array}{l}\text { SIGAPS } \\
27 \text { CHU }\end{array}$ & Part (\%) & \\
\hline Global & 3208248 & 68892 & 2,15 & 4206147 & 81900 & 1,95 & $-9,3$ \\
\hline Alzheimer & 47841 & 1277 & 2,67 & 70340 & 1972 & 2,80 & $+5,0$ \\
\hline AVC & 27985 & 870 & 3,11 & 36696 & 1178 & 3,21 & $+3,3$ \\
\hline Parkinson & 11002 & 555 & 5,04 & 14837 & 652 & 4,39 & $-12,9$ \\
\hline Diabète & 54648 & 1511 & 2,76 & 70695 & 1741 & 2,46 & $-10,9$ \\
\hline SIDA & 34199 & 1741 & 5,09 & 41879 & 1766 & 4,22 & $-17,2$ \\
\hline
\end{tabular}


Tableau III : Evolution du nombre d'articles originaux, score SIGAPS et pourcentage d'articles classés A et B entre 2004-2008 (P1) et 2009-2013 (P2) pour la maladie d'Alzheimer, les Accidents Vasculaires Cérébraux (AVC), la maladie de Parkinson, le diabète et le SIDA.

\begin{tabular}{|c|c|c|c|c|c|c|c|c|c|c|}
\hline & \multicolumn{2}{|c|}{ Alzheimer } & \multicolumn{2}{|c|}{ AVC } & \multicolumn{2}{|c|}{ Parkinson } & \multicolumn{2}{|c|}{ Diabète } & \multicolumn{2}{|c|}{ SIDA } \\
\hline & $\begin{array}{l}2004 \\
2008\end{array}$ & $\begin{array}{l}2009 \\
2013 \\
\end{array}$ & $\begin{array}{l}2004 \\
2008\end{array}$ & $\begin{array}{l}2009 \\
2013\end{array}$ & $\begin{array}{l}2004 \\
2008\end{array}$ & $\begin{array}{l}2009 \\
2013\end{array}$ & $\begin{array}{l}2004 \\
2008 \\
\end{array}$ & $\begin{array}{l}2009 \\
2013\end{array}$ & $\begin{array}{l}2004 \\
2008\end{array}$ & $\begin{array}{l}2009 \\
2013 \\
\end{array}$ \\
\hline Nb Articles & 1277 & 1972 & 870 & 1179 & 555 & 652 & 1507 & 1741 & 1741 & 1766 \\
\hline Evol (\%) & \multicolumn{2}{|c|}{$+54,5$} & \multicolumn{2}{|c|}{$+35,5$} & \multicolumn{2}{|c|}{$+17,5$} & \multicolumn{2}{|c|}{$+15,5$} & \multicolumn{2}{|c|}{$+1,4$} \\
\hline \multirow{2}{*}{$\begin{array}{l}\text { Score } \\
\text { Evol (\%) }\end{array}$} & 18038 & 29306 & 13627 & 19874 & 9375 & 11160 & 21013 & 25490 & 29002 & 30222 \\
\hline & \multicolumn{2}{|c|}{$+62,5$} & \multicolumn{2}{|c|}{$+45,8$} & \multicolumn{2}{|c|}{$+19,0$} & \multicolumn{2}{|c|}{$+21,3$} & \multicolumn{2}{|c|}{$+4,2$} \\
\hline$\% \mathrm{AB}$ & 33,7 & 42,9 & 39,8 & 51,9 & 55,7 & 55,5 & 33,6 & 42,6 & 50,1 & 57,8 \\
\hline Evol (\%) & \multicolumn{2}{|c|}{$+27,3$} & \multicolumn{2}{|c|}{$+30,4$} & \multicolumn{2}{|c|}{$-0,4$} & \multicolumn{2}{|c|}{$+26,8$} & \multicolumn{2}{|c|}{$+15,4$} \\
\hline
\end{tabular}

Tableau IV: Indices de Spécialisation (IS) de la production scientifique des 7 inters régions françaises, sur 5 pathologies de 2004 à 2008 et 2009 à 2013. En jaune, les IS < 0,775 ( $1^{\text {er }}$ Quartile), en vert les IS > 1,23 ( ${ }^{\text {ème }}$ Quartile).

\begin{tabular}{|c|c|c|c|c|c|c|c|c|c|c|}
\hline & \multicolumn{10}{|c|}{ Indices de Spécialisation (Scores) } \\
\hline & \multicolumn{2}{|c|}{ Alzheimer } & \multicolumn{2}{|c|}{ AVC } & \multicolumn{2}{|c|}{ Parkinson } & \multicolumn{2}{|c|}{ Diabète } & \multicolumn{2}{|c|}{ SIDA } \\
\hline & $\begin{array}{c}2004- \\
2008 \\
\end{array}$ & $\begin{array}{l}2009- \\
2013 \\
\end{array}$ & $\begin{array}{c}2004- \\
2008 \\
\end{array}$ & $\begin{array}{c}2009- \\
2013 \\
\end{array}$ & $\begin{array}{c}2004- \\
2008 \\
\end{array}$ & $\begin{array}{c}2009- \\
2013 \\
\end{array}$ & $\begin{array}{c}2004- \\
2008 \\
\end{array}$ & $\begin{array}{l}2009- \\
2013 \\
\end{array}$ & $\begin{array}{c}2004- \\
2008 \\
\end{array}$ & $\begin{array}{r}2009- \\
2013 \\
\end{array}$ \\
\hline Est & 0,83 & 0,94 & 0,93 & 1,13 & 0,31 & 0,36 & 0,97 & 1,17 & 0,44 & 0,54 \\
\hline Grand Ouest & 0,81 & 0,92 & 0,52 & 0,62 & 1,02 & 1,11 & 0,98 & 1,05 & 0,75 & 0,79 \\
\hline Ile de France & 0,85 & 0,81 & 1,05 & 1,04 & 0,80 & 0,75 & 1,06 & 1,00 & 1,43 & 1,51 \\
\hline Nord Ouest & 1,73 & 1,54 & 1,75 & 1,60 & 1,81 & 1,46 & 0,95 & 1,25 & 0,32 & 0,47 \\
\hline Rhône AA & 0,67 & 0,77 & 1,07 & 0,97 & 1,54 & 1,48 & 0,94 & 0,94 & 0,52 & 0,60 \\
\hline Sud Méd & 1,26 & 1,08 & 0,43 & 0,60 & 0,51 & 0,69 & 0,91 & 0,87 & 1,03 & 1,03 \\
\hline SOOM & 1,94 & 2,19 & 1,07 & 1,08 & 1,96 & 2,36 & 1,01 & 0,98 & 1,75 & 1,68 \\
\hline
\end{tabular}

Rhône AA : Rhône Alpes Auvergne ; Sud Méd : Sud Méditerranée ; SOOM : Sud-Ouest Outre Mer 
Annexe 1 : Requêtes utilisées dans la base PubMed pour délimiter le périmètre de recherche pour chaque pathologie

\begin{tabular}{|c|c|}
\hline Pathologie & Requête Pubmed \\
\hline Global & "journal article"[Publication Type] \\
\hline Alzheimer & $\begin{array}{l}\text { ((alzheimer*) OR (Fronto-temporal dementia) OR (semantic dementia) OR (posterior cortical atrophy) OR } \\
\text { (progressive aphasia) OR (brain aging) OR (dementia) OR (lewy disease) OR (cognitive impairment) OR } \\
\text { (cognitive decline) OR (beta*amyloid OR AMYLOID*BETA OR (amyloid precursor)) OR (APP and } \\
\text { processing) OR (presenilin*) OR (ApoE and (brain or neurology)) OR (tau protein) NOT (Parkinson } \\
\text { Disease[Mesh Terms] OR Huntington Disease[Mesh Terms] OR Creutzfeldt-Jakob Syndrome[Mesh Terms] OR } \\
\text { CADASIL[Mesh Terms] OR Amyotrophic Lateral Sclerosis[Mesh Terms] OR Parkinsonian Disorders[Mesh } \\
\text { terms])) AND "journal article"[Publication Type] }\end{array}$ \\
\hline AVC & (Stroke[MeSH Terms] OR "Brain Ischemia"[MeSH Terms]) AND "journal article"[Publication Type] \\
\hline Parkinson & 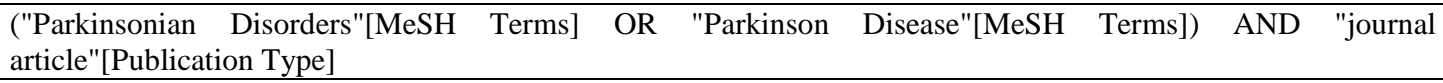 \\
\hline Diabète & Diabetes Mellitus[MeSH Terms] AND "journal article"[Publication Type] \\
\hline SIDA & HIV Infections[MeSH Terms] AND "journal article"[Publication Type] \\
\hline
\end{tabular}


Figure 1 : Evolution quantitative et qualitative du nombre d'articles originaux sur la maladie d'Alzheimer publiés par les CHU de 2004 à 2013 (données SIGAPS)

Catégorie A : $10 \%$ des revues ayant les Facteurs d'Impact (FI) les plus élevés et catégorie B, $15 \%$ des revues suivantes. Catégories C, D et E, quartiles suivants par FI décroissants. Catégorie NC (Non Classées) : revues non référencées n'ayant pas de FI.

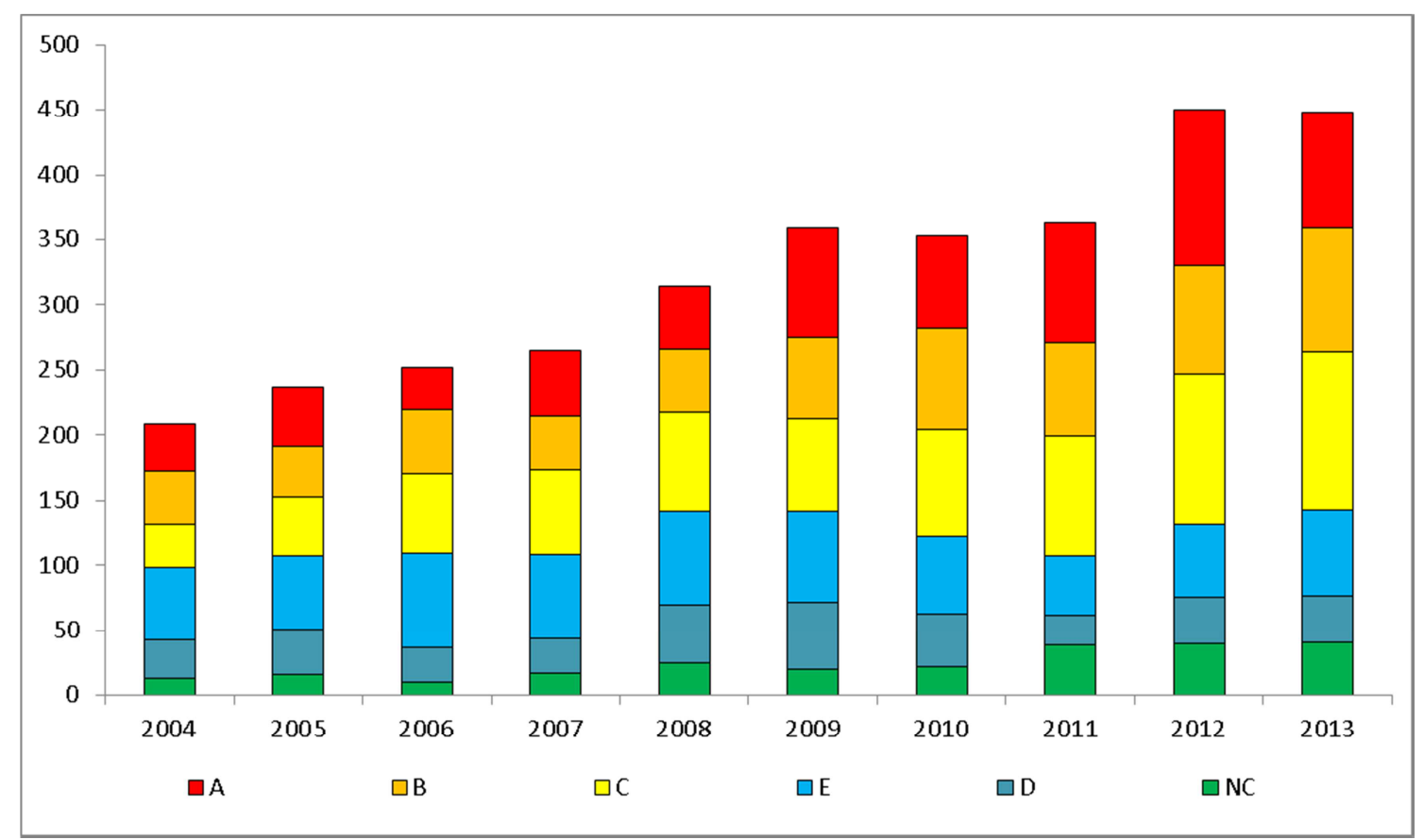


Figure 2: Indices de Spécialisation (IS) de la production scientifique des inters régions françaises sur 5 pathologies de 2004 à 2008 et 2009 à 2013. En bleu, l'IS sur la période 2004-2008, en jaune sur la période 2009-2013.

1 : GIRCI Est

2 : GIRCI Grand Ouest

3 : GIRCI Ile de France

4 : GIRCI Nord-Ouest

5 : GIRCI Rhône Alpes-Auvergne

6 : GIRCI Sud Méditerranée

7 : GIRCI Sud-Ouest Outre-Mer
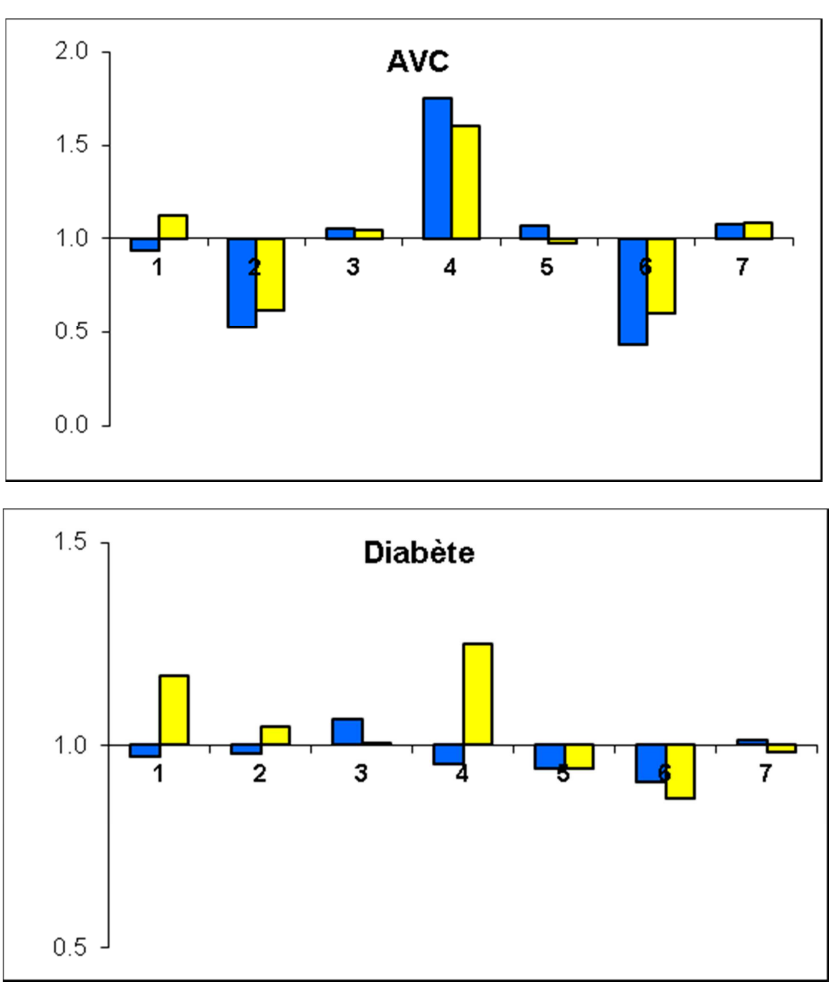
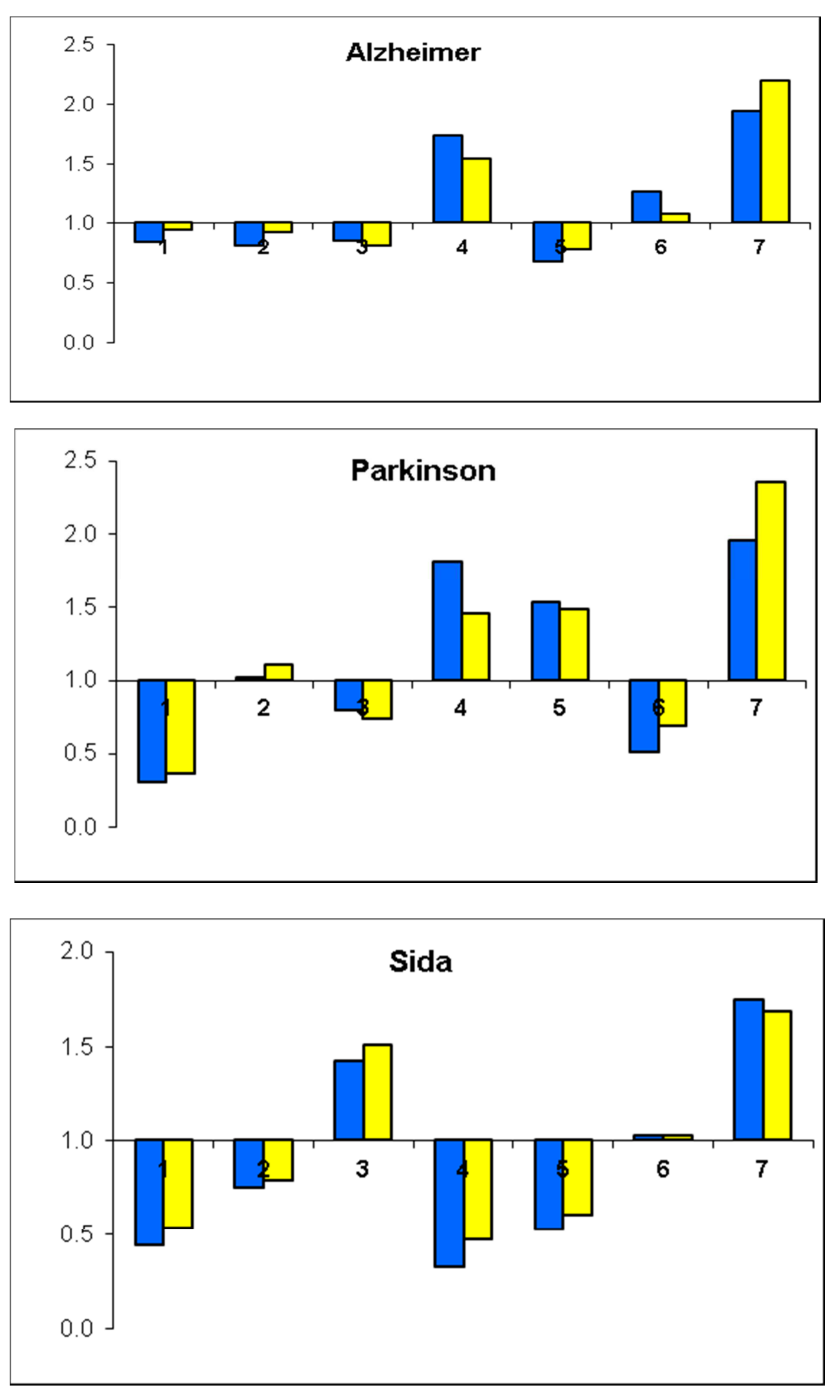
Figure 3 : Comparaison des Indices de Spécialisation (IS) «Alzheimer » des CHU métropolitains entre les 2 périodes 2004-2008 et 2009-2013. La taille de la bulle est proportionnelle au score SIGAPS global des publications sur la maladie d'Alzheimer sur la période 2004-2013 (médiane et [Min - Max])

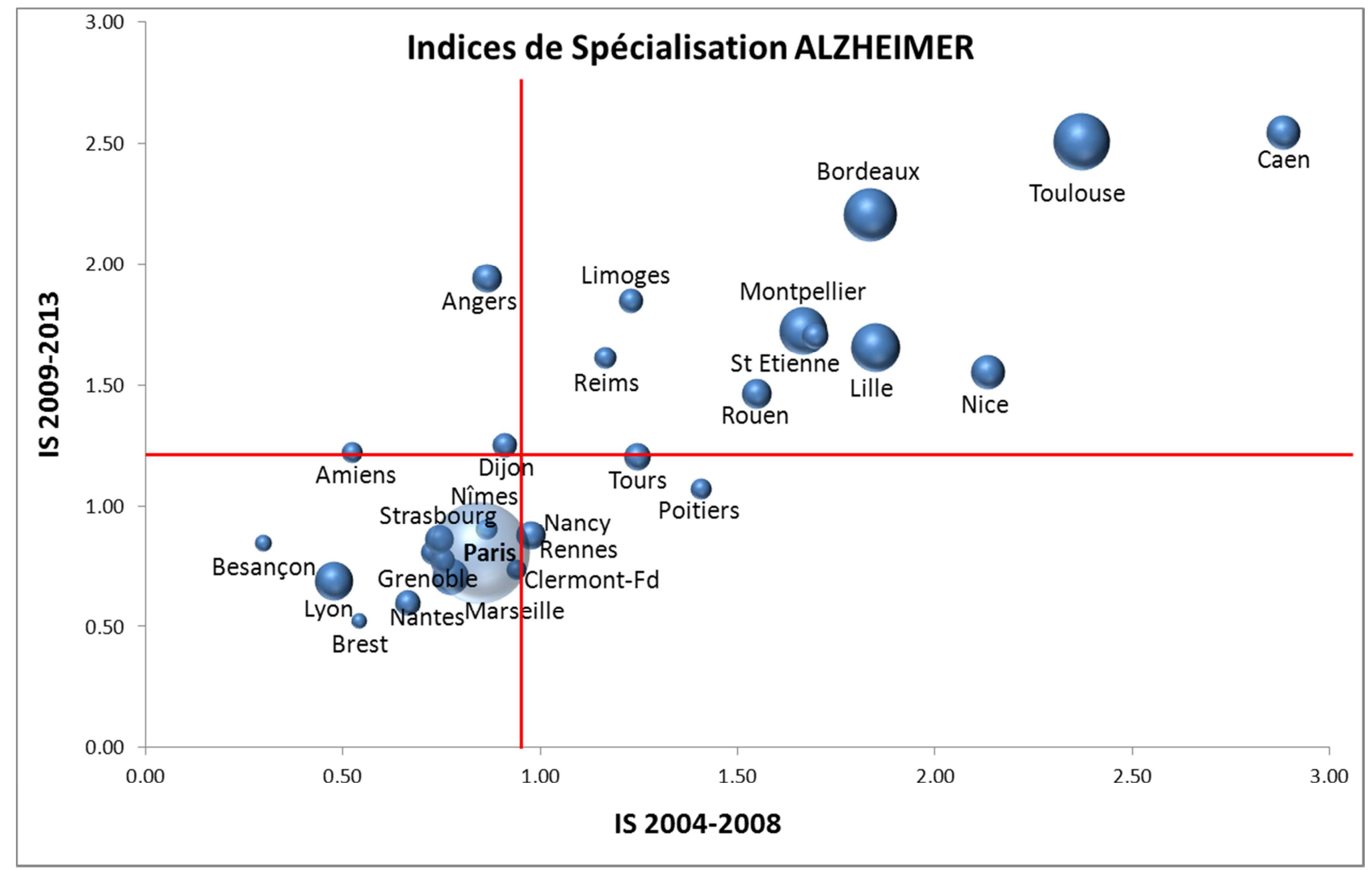

IS médian 2004-2008 : 0,94 [0,30 - 2,88]

IS médian 2009-2013: 1,21 [0,53 - 2,55]

Corrélation (Spearman) $: r=0,79$ 
Figure 4: Comparaison des Indices de Spécialisation (IS) AVC, maladie de Parkinson, diabète et SIDA des CHU métropolitains entre les 2 périodes 2004-2008 et 2009-2013. La taille de la bulle est proportionnelle au score SIGAPS global des publications sur chacune de ces maladies sur la période 2004-2013.

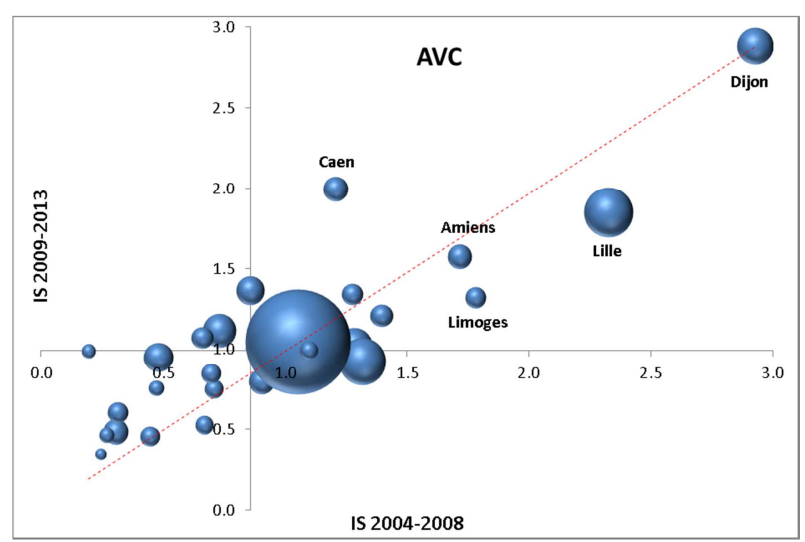

IS médian 2004-2008 = 0,86 [0,19-2,86]

IS médian 2009-2013 = 0,99 $0,35-2,89]$

Corrélation : $r=0,79$

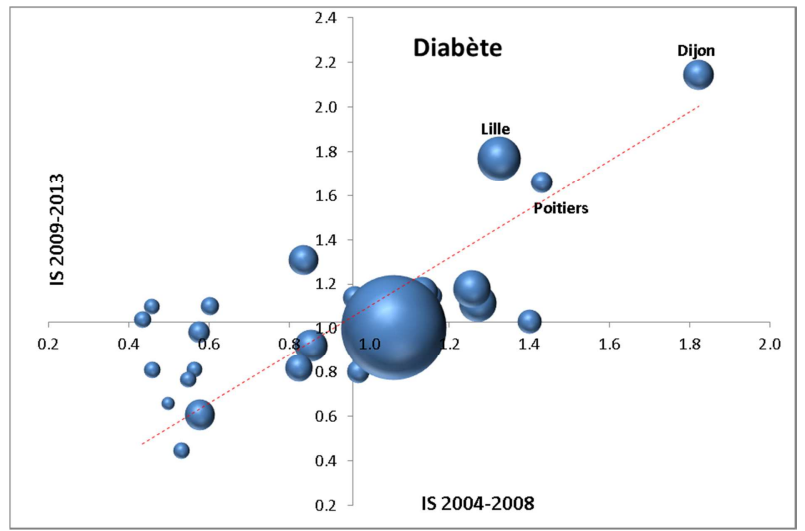

IS médian 2004-2008 = 0,96 [0,43-1,82]

IS médian 2009-2013 = 1,03 [0,45 - 2,15]

Corrélation : $r=0,67$

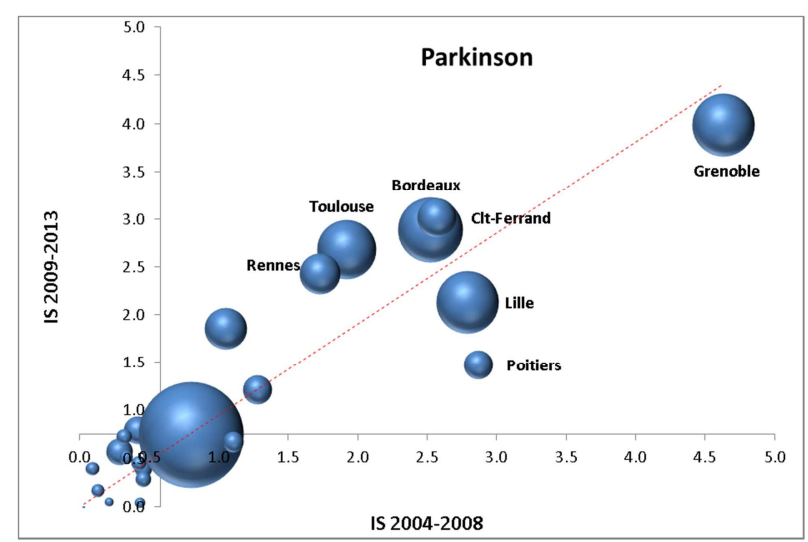

IS médian 2004-2008 = 0,58 [0,03 - 4,63]

IS médian 2009-2013 = 0,75 [0,00 - 3,99]

Corrélation : $r=0,85$

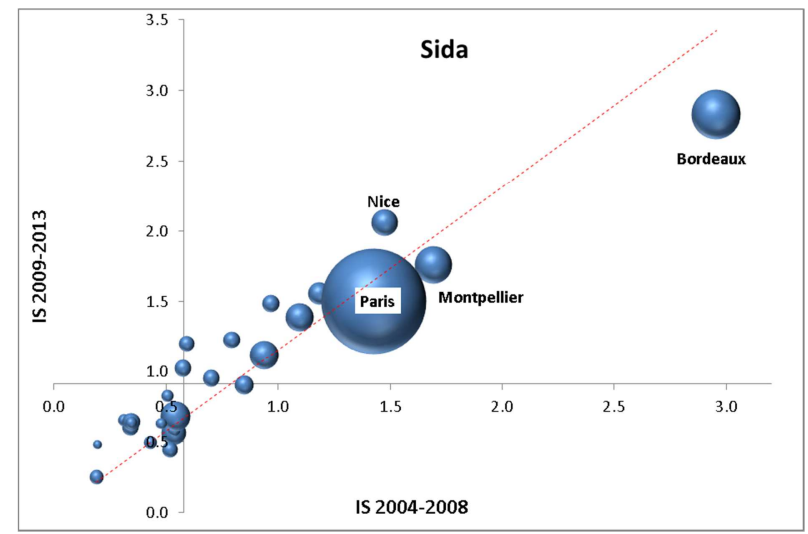

IS médian 2004-2008 = 0,58 [0,19-2,95]

IS médian 2009-2013 = 0,91 [0,25 - 2,83]

Corrélation : $r=0,90$ 